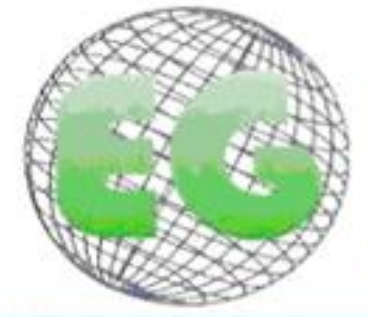

No38

\title{
La red social del adolescente: la influencia de la amistad en el desarrollo de hábitos obesogénicos
}

The social network of adolescents: The influence of friendship in the development of obesogenic habits

\section{*Arias Ramos, Natalia **Marqués Sánchez, María Pilar ${ }^{* * *}$ Calvo Sánchez, María Dolores ***'Sánchez García, Ana Belén *Quiroga Sánchez, Enedina *García López, Rosa María}

\author{
*Hospital de El Bierzo. Universidad de León. SALBIS Research Group. E-mail: narir@unileon.es \\ **Universidad de León. SALBIS Research Group ***Universidad de Salamanca. **** Hospital General \\ Universitario Reina Sofía de Murcia. Universidad de Murcia. España.
}

Palabras clave: Obesidad; Adolescente; Redes sociales; Amigos; Hábitos alimenticios; Ejercicio físico Keywords: obesity; adolescent; social networks; peers; food intake; physicaly activity

\section{RESUMEN}

El presente artículo pretende justificar la relevancia de las relaciones sociales en el entorno del adolescente obeso. La motivación por este enfoque surgió debido al vacío que se ha encontrado en estudios que expliquen si los adolescentes comen mejor o peor y/o realizan más o menos ejercicio físico dependiendo de su red de amigos. Se realizó una búsqueda bibliográfica centrada en la obesidad y el análisis de las redes sociales de los jóvenes a través de la Biblioteca de la Universidad de León, en diferentes bases de datos tales como Web of Knowledge (WOK), Scielo, Scopus, entre otras. Se obtuvieron artículos y otros documentos de interés utilizando revistas pertenecientes a las disciplinas de la Sociología y de las Ciencias de la Salud. Tras realizar la lectura de los textos más relevantes se pudo obtener los siguientes resultados: (1) las relaciones influyen en los hábitos de los adolescentes, (2) la calidad y la cantidad de comida puede depender del tipo de red que le rodea, y (3) el nivel de ejercicio físico que tiene un individuo se puede ver influenciado por sus mejores amigos, por el grupo en general que le rodea e incluso por los amigos de sus amigos. De todo ello se deriva, que el estudio de la red social del individuo puede ser una herramienta útil a la hora de visualizar los patrones de comportamiento, y por tanto, aplicable a los programas de salud y salud pública contribuyendo, de esta forma, a la sostenibilidad del sistema.

\section{ABSTRACT}

This aim of this article is to demonstrate the importance of social networks in the obese adolescent's environment. This study emerged in response to the paucity of information encountered in the literature as regards whether adolescents eat better or worse and/or performs more or less physical exercise 
depending on their friendship networks. We performed a literature search at the University of León library which focused on obesity and an analysis of young people's social networks, exploring databases such as the Web of Knowledge (WOK), SciELO and Scopus, among others. Articles and other documents of interest were identified using journals from the disciplines of Sociology and the Health Sciences. After the most relevant texts had been read in full, the following results were obtained: (1) relationships influence adolescents' habits, (2) the quality and quantity of food they consume can depend on the type of network to which they belong, and (3) the amount of physical exercise that adolescents do can be influenced by their best friends, by the wider group to which they belong and even by friends of friends. The foregoing suggests that the study of adolescents' social networks could be a useful tool for identifying behaviour patterns; it is therefore applicable to health and public health programmes and would contribute to the sustainability of the system.

\section{INTRODUCCIÓN}

El estudio de todos los factores que pueden contribuir a la ganancia de peso, entre los que se incluye la influencia social, ha cobrado una especial importancia, ya que la obesidad infanto-juvenil ha sido considerada por la Organización Mundial de la Salud (OMS), como uno de los problemas de salud pública de mayor calado del siglo XXI ${ }^{(1)}$.

El inicio de este trastorno nutricional en edades tan tempranas es preocupante, al demostrarse que los niños y adolescentes afectados por el exceso de peso, mantienen con una mayor probabilidad esta condición hasta la edad adulta ${ }^{(2)}$. Esta situación impacta directamente sobre la calidad de vida del individuo elevando el riesgo de padecer afecciones a corto y a largo plazo que aumentan la morbimortalidad y el gasto sanitario, tales como la diabetes tipo II, enfermedades cardiovasculares, trastornos del aparato locomotor y ciertos tipos de cáncer, así como otras afectaciones psicológicas como la ansiedad y la depresión ${ }^{(3)}$.

Las elevadas cifras de prevalencia de las que informan distintos países de todo el mundo han hecho que se desarrollen estrategias de diversos tipos que hasta el momento no han dado los resultados esperados. Esta situación ha provocado que instituciones y organismos competentes en esta materia lleven a cabo nuevos planteamientos que tengan por objetivo la disminución o el estancamiento de esta epidemia, creando y fomentando políticas duraderas en el tiempo, en las que se involucren a todos los agentes implicados en la lucha contra la obesidad infantojuvenil (4). En consecuencia, Europa con sus nuevos programas marcos en investigación y desarrollo ya recoge esta preocupación por la obesidad y el sobrepeso, así como por otras enfermedades derivadas de una carencia de hábitos saludables. Tal es el caso del propio Programa Horizon 2020, en el cual se pretende, a través de sus tres pilares básicos, abordar los problemas más importantes para la población en general. En concreto, y de interés para el presente estudio, dentro del pilar denominado Retos Sociales, se ha creado la estrategia denominada "salud, cambio demográfico y bienestar", cuyo objetivo es promocionar la salud y el bienestar de todos, y mejorar la prevención de las enfermedades, incluyéndose aquí el problema de la obesidad y el sobrepeso ${ }^{(5)}$.

Para desarrollar esta revisión bibliográfica, donde el estudio de las redes sociales del adolescente y su influencia en determinados hábitos relacionados con la obesidad es el punto más importante, se procederá en primer lugar a explicar por qué el exceso de peso en la adolescencia resulta un problema que responde a la influencia social. Seguidamente, se expondrá en qué consiste el método del Análisis de Redes Sociales (ARS), con el que se podrían cuantificar las relaciones de los jóvenes y así poder facilitarnos información sobre patrones de comportamiento social que favorecerían o no, la obesidad. Por último, se expondrá una serie de estudios donde 
se ha comprobado cómo el entorno social del adolescente tiene gran capacidad de influencia en la ingesta alimenticia y en la realización de ejercicio físico así como en comportamiento sedentario.

\section{El exceso de peso en la adolescencia, un problema con gran influencia social}

El sobrepeso y la obesidad en adultos y en niños son considerados como un gran problema multifactorial en el que no solo la genética y las características propias del individuo tienen la respuesta para poder obtener una solución ${ }^{(2)}$. Parece que el medio social que nos rodea ha tomado especial relevancia a la hora de adquirir y mantener en el tiempo actitudes y comportamientos transcendentales en las condiciones de salud de la persona ${ }^{(6)}$. El estudio de Cristakis y Fowler supuso un antes y un después en esta afirmación puesto que estos investigadores lograron demostrar en adultos que la probabilidad de convertirse en obeso es de un $57 \%$ si un amigo lo es entre otros hallazgos de interés ${ }^{(6)}$.

La adolescencia supone en este sentido un periodo especial a tener en cuenta, es el periodo de transición a la edad adulta en la que se suceden adaptaciones fisiológicas, psicológicas y sociales que pueden poner en peligro hábitos saludables adquiridos en la infancia ${ }^{(7)}$. Además, se suma a este hecho la falta de sensación de peligro y la ausencia de percepción de consecuencias en la realización de sus comportamientos $^{\left({ }^{8}\right)}$. Es, por tanto, un periodo complejo en el que se desarrolla el autoconcepto y la autoestima ${ }^{(7)}$, con multitud de influencias procedentes de su red de amigos y de la sociedad en general que forma parte de su vida y con la que contacta de forma constante. De esta forma, las conductas de los adolescentes podrían responder a un patrón similar al de las relaciones que los rodean, o lo que es lo mismo, a su red social, de ahí la importancia del estudio y análisis de la red para el fomento de comportamientos saludables ${ }^{(9)}$.

La obesidad se contagia de forma epidémica por la red social del individuo ${ }^{(6)}$ y la forma en la que se propaga en individuos de cualquier edad ha supuesto motivo de estudio desde un punto de vista psicológico. Parece que cada vez más, el sobrepeso y la obesidad han pasado a ser algo "normal" en niños, adolescentes y adultos, favoreciendo la iniciación y mantenimiento de ciertos comportamientos que favorecen la ganancia de peso. Se ha estudiado si los hábitos obesogénicos son causa de la imitación entre iguales o en cambio, si son provocados y/o estimulados por motivos de facilitación social o también por una cuestión de imposición de normas sociales, sin llegar a una conclusión definitiva ${ }^{(10)}$.

Este planteamiento de la base psicológica como causa de determinados comportamientos nos lleva a pensar que el entorno social tiene un peso todavía poco explorado a nivel científico. La necesidad de pertenencia a un grupo y el miedo a una estigmatización social, sobre todo en el periodo de la adolescencia, hacen que se modifiquen hábitos y comportamientos que hasta el momento estaban bajo el control de los progenitores, resaltando de esta forma la importancia de las relaciones sociales en la vida del joven, aspecto de interés para el presente estudio.

\section{El Análisis de Redes Sociales (ARS) y su aplicación en el campo de la salud}

La red social del individuo se comporta como una estructura dinámica que condiciona su comportamiento en varios aspectos de la vida. Se define red social como un conjunto de nodos y de lazos, representando éstos últimos la existencia o la carencia 
de alguna relación entre los nodos ${ }^{(11)}$. La red implica un tejido de relaciones a través de las cuales se transfieren recursos como información, ayuda y consejo siendo necesario para que ello sea posible, establecer vínculos de confianza y de amistad que faciliten alcanzar el objetivo entre los actores o nodos implicados ${ }^{(9)}$. Por tal motivo, parece relevante estudiar qué tipo de recursos se transfieren a través de los vínculos de la amistad, utilizando para ello el ARS.

El ARS ${ }^{(12)}$ es un método que cuantifica las relaciones y nos permite comprender la dinámica de contactos que se establecen entre los actores. Esta metodología está basada en la Teoría de Grafos, en la que además otras disciplinas como la antropología, la sociología o la psicología han jugado un papel fundamental en la interpretación de los datos relacionales de los individuos que conforman la red. Este tipo de análisis aporta información sobre las características de la estructura reticular a la que pertenecen los individuos, tales como el tamaño de la red, los subgrupos que se forman (clusters), el grado de relaciones o vínculos (degree), el grado de intermediación (betweenness) y de cercanía de los nodos (closeness), así como el grado de influencia en la adquisición de determinados comportamientos (eigenvector). En definitiva, con el ARS se miden cuantitativamente aspectos, que a veces, pueden resultar invisibles.

El ARS ya ha sido aplicado al campo de la salud, demostrando cómo las relaciones condicionan varios procesos patológicos ${ }^{(9)}$. Por ejemplo, hay estudios en donde se ha podido constatar cómo la red social favorece la velocidad de propagación de una enfermedad ${ }^{(13)}$, la influencia en determinados procesos contagiosos ${ }^{(14)}$, el riesgo de contraer VIH ${ }^{(15)}$, los hábitos para mejorar la salud cardiovascular ${ }^{(16)}$, etc. De todo ello se deriva que el entorno social influye, incluso, en determinadas enfermedades convertidas en epidemias, por lo que lo mismo podría ocurrir en el caso de la obesidad.

En este sentido, ya se ha podido constatar que el adolescente con elevado índice de masa corporal tiene unas determinadas características en su patrón de relaciones dentro de la red $\left(17,{ }^{18}\right)$, tales como una posición periférica y un bajo grado de relaciones, algo que no es precisamente similar a lo que ocurre en estudios de fumadores $\mathrm{y} / \mathrm{o}$ consumidores de alcohol. Tiende a escoger como amigo a aquellos nodos con los que comparte la característica del sobrepeso. En consecuencia, se podría obtener como conclusión que el adolescente obeso tendría menos recursos relacionales para poder afrontar el objetivo de perder peso. Esta predisposición a elegir individuos similares como amigos, es lo que en términos sociológicos se define como Homofilia ${ }^{(19)}$. Este concepto hace referencia al hecho por el cual el individuo establece sus relaciones atendiendo a aspectos de semejanza relacionados con la edad, el género, determinados hábitos, formas de pensar u otras características distintivas, marcando los procesos de selección social en todo tipo de edades. En definitiva, a través de procesos como el de la homofilia, el adolescente establece lazos con otros individuos afines, formando una red, cuyo análisis y estudio podría aportarnos datos útiles para poder modificar hábitos perjudiciales para su salud.

\section{MATERIAL Y MÉTODO}

Para llevar a cabo este trabajo se ha realizado una búsqueda específica de documentos tales como estudios de investigación tanto transversales como longitudinales, revisiones bibliográficas publicadas sobre todo en idioma anglosajón y otros documentos relevantes en los que el Análisis de Redes Sociales y la obesidad 
infantojuvenil era el aspecto más relevante. La búsqueda de la documentación se llevó a cabo a través de la biblioteca de la Universidad de León, utilizando las siguientes bases de datos: Web of knowledge (WOK), Scielo, Scopus, entre otras. A su vez las revistas más consultadas pertenecían a disciplinas variadas como la Pediatría, la Psicología, la Salud Pública y las Ciencias Sociales.

Para la búsqueda en las diferentes plataformas se utilizaron una serie de palabras clave tanto en inglés como en castellano, siendo las más utilizadas: obesidad (obesity), adolescente (adolescent), redes sociales (social networks), amigos (peers), hábitos alimenticios (food intake), ejercicio físico (physicaly activity) y comportamiento sedentario (sedentary behavior). La obtención de los artículos y documentos màs relevantes que figuran en esta revisión se realizó durante los meses de abril y mayo de 2014, con el fin de ofrecer en esta publicación la literatura más reciente. Para afinar la búsqueda solo se utilizaron aquellos documentos a texto completo publicados desde el año 2000 hasta la fecha, artículos usando de forma puntual textos o documentos más antiguos que nos sirven de referencia por su especial relevancia.

\section{RESULTADOS}

A continuación, se exponen bajo este epígrafe los resultados más relevantes encontrados en artículos de especial interés utilizados en esta revisión bibliográfica, donde se ha comprobado cómo el entorno social del joven tiene gran capacidad de influencia en comportamientos relacionados con la salud y más concretamente con la obesidad, como son la ingesta alimenticia, la realización de ejercicio físico y los hábitos sedentarios.

A modo esquemático se muestran las principales variables estudiadas así como los resultados de mayor importancia obtenidos por los autores. Cabe destacar que los resultados aquí expuestos son de estudios realizados con población adolescente, de interés en este documento, excluyendo de esta forma muestras infantiles y adultas.

Tabla I. Trabajos empíricos en los que se pone de manifiesto la influencia de la red social en la alimentación y el ejercicio físico de los adolescentes.

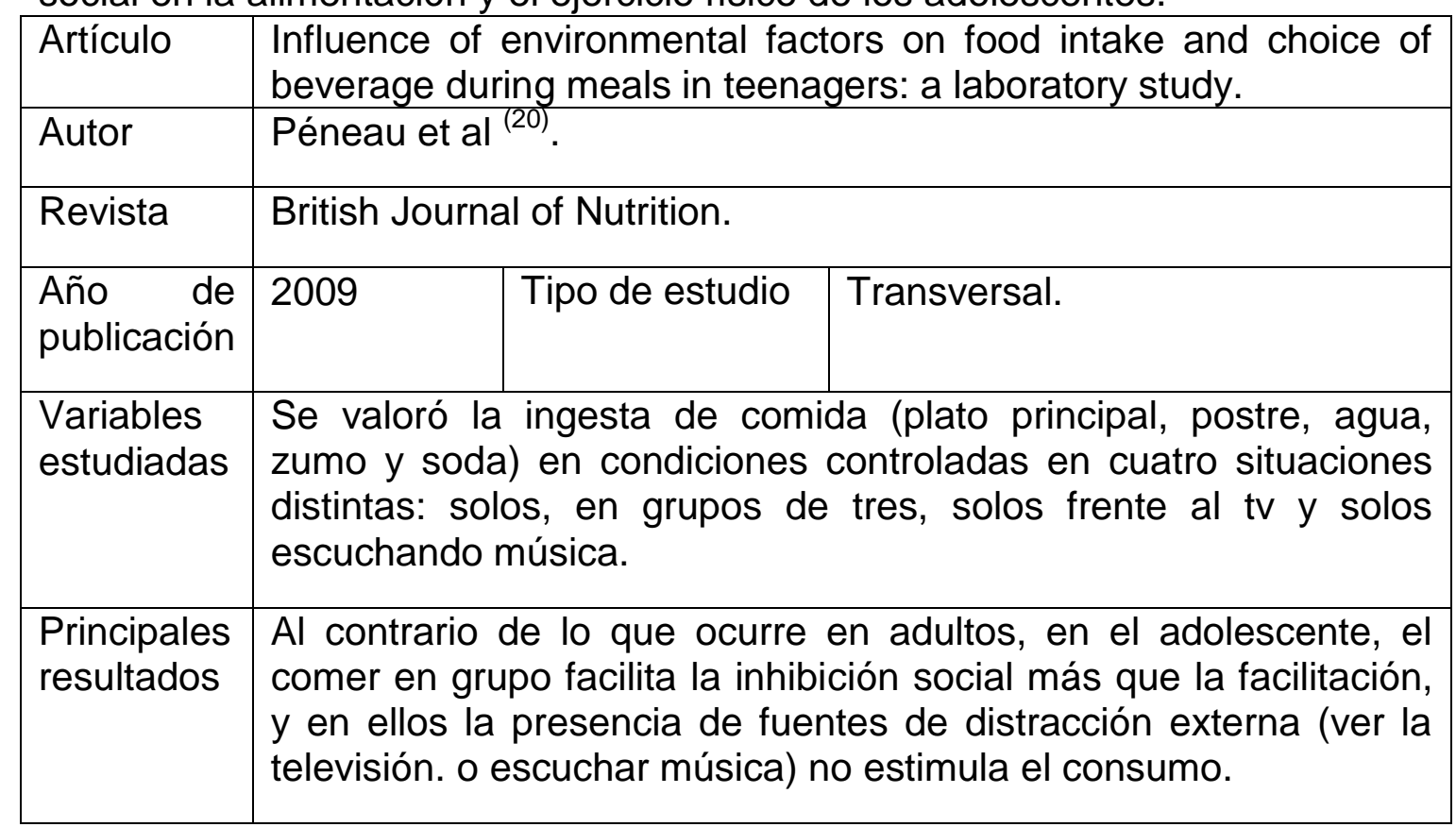




\begin{tabular}{|c|c|c|}
\hline Artículo & \multicolumn{2}{|c|}{ Obesity-related behaviors in adolescent friendship networks. } \\
\hline Autor & \multicolumn{2}{|l|}{ De la Haye et al ${ }^{(21)}$. } \\
\hline Revista & \multicolumn{2}{|l|}{ Social Networks. } \\
\hline $\begin{array}{l}\text { Año de } \\
\text { publicación }\end{array}$ & Tipo de estudio & Transversal. \\
\hline $\begin{array}{l}\text { Variables } \\
\text { estudiadas }\end{array}$ & \multicolumn{2}{|c|}{$\begin{array}{l}\text { Se evalúo la frecuencia de ingesta de determinada comida } \\
\text { altamente calórica, la realización (en cantidad y frecuencia) de } \\
\text { actividad física, el tiempo utilizado frente a dispositivos de pantalla } \\
\text { y los lazos de amistad con sus compañeros de clase. También el } \\
\text { IMC (Índice de masa corporal). }\end{array}$} \\
\hline $\begin{array}{l}\text { Principales } \\
\text { resultados }\end{array}$ & \multicolumn{2}{|c|}{$\begin{array}{l}\text { Dentro de los grupos formados del mismo sexo, se encontró } \\
\text { similitud en la realización de ejercicio físico organizado. En el } \\
\text { análisis de los grupos de amistades por sexos, los conjuntos } \\
\text { femeninos participaron de forma similar en actividades de } \\
\text { pantalla. En cambio, los grupos de amigos masculinos lo hicieron } \\
\text { en el consumo de comida altamente calórica. Con respecto a } \\
\text { papel que ocupa el alumno/a popular dentro de las redes } \\
\text { formadas en la escuela, se pudo comprobar su influencia en } \\
\text { algunos de los comportamientos saludables. }\end{array}$} \\
\hline
\end{tabular}

\begin{tabular}{|c|c|c|}
\hline Artículo & \multicolumn{2}{|c|}{$\begin{array}{l}\text { Weight-related behavior among adolescents: the role of peer } \\
\text { effects. }\end{array}$} \\
\hline Autor & \multicolumn{2}{|c|}{ Ali et al ${ }^{(22)}$} \\
\hline Revista & \multicolumn{2}{|l|}{ Plos One. } \\
\hline $\begin{array}{l}\text { Año de } \\
\text { publicación }\end{array}$ & Tipo de estudio & Longitudinal. \\
\hline $\begin{array}{l}\text { Variables } \\
\text { estudiadas }\end{array}$ & \multicolumn{2}{|c|}{$\begin{array}{l}\text { Se tuvo en cuenta la realización de actividad física regular así } \\
\text { como la práctica de deportes activos. También las horas que el } \\
\text { adolescente ve la tv/vídeo, y el tiempo dedicado al sueño. Con } \\
\text { respecto a los hábitos alimenticios se preguntó sobre el desayuno, } \\
\text { la frecuencia de consumo en restaurantes de comida rápida, la } \\
\text { ingesta de frutas o vegetales diariamente, el consumo de snacks } \\
\text { calóricos y por último, el IMC. }\end{array}$} \\
\hline $\begin{array}{l}\text { Principales } \\
\text { resultados }\end{array}$ & \multicolumn{2}{|c|}{$\begin{array}{l}\text { Se encontró evidencia significativa de que los amigos influían en } \\
\text { la realización de deportes y actividad física regular así como en la } \\
\text { frecuencia de consumo de comida rápida. En cambio no se } \\
\text { obtuvo evidencia de influencia en ver la televisión, las horas de } \\
\text { sueño, el desayuno y el consumo de snacks, frutas y vegetales. }\end{array}$} \\
\hline
\end{tabular}




\begin{tabular}{|c|c|c|}
\hline Artículo & \multicolumn{2}{|c|}{$\begin{array}{l}\text { Relationship between adolescents' and their friends' eating } \\
\text { behaviors: breakfast, fruit, vegetable, whole-Grain, and dairy } \\
\text { intake. }\end{array}$} \\
\hline Autor & \multicolumn{2}{|c|}{ Bruening et al ${ }^{(23)}$} \\
\hline Revista & \multicolumn{2}{|c|}{ Journal of the academy of nutrition and dietetics. } \\
\hline $\begin{array}{l}\text { Año de } \\
\text { publicación }\end{array}$ & Tipo de estudio & Transversal. \\
\hline $\begin{array}{l}\text { Variables } \\
\text { estudiadas }\end{array}$ & \multicolumn{2}{|c|}{$\begin{array}{l}\text { Se relacionó el consumo de determinados alimentos por parte de } \\
\text { los adolescentes (desayuno, frutas y verduras, cereales de grano } \\
\text { entero y productos lácteos) con la ingesta de los mismos } \\
\text { alimentos por parte del grupo de sus mejores amigos y del grupo } \\
\text { de amigos o compañeros. }\end{array}$} \\
\hline $\begin{array}{l}\text { Principales } \\
\text { resultados }\end{array}$ & \multicolumn{2}{|c|}{$\begin{array}{l}\text { Se encontró una asociación positiva en la ingesta del desayuno, } \\
\text { de cereales de grano entero y de productos lácteos entre los } \\
\text { adolescentes y ambos grupos de amigos. En cambio en los } \\
\text { vegetales se encontró asociación únicamente con sus mejores } \\
\text { amigos siendo nula en el consumo de fruta. }\end{array}$} \\
\hline
\end{tabular}

\begin{tabular}{|c|c|c|c|}
\hline Artículo & \multicolumn{3}{|c|}{$\begin{array}{l}\text { The association of objectively determined physical activity behavior } \\
\text { among adolescent female friends. }\end{array}$} \\
\hline Autor & \multicolumn{3}{|c|}{ Schofield et al ${ }^{(24)}$} \\
\hline Revista & \multicolumn{3}{|c|}{ Research Quarterly for Exercise and Sport. } \\
\hline $\begin{array}{l}\text { Año de } \\
\text { publicación }\end{array}$ & 2007 & Tipo de estudio & Tranversal \\
\hline $\begin{array}{l}\text { Variables } \\
\text { estudiadas }\end{array}$ & \multicolumn{3}{|c|}{$\begin{array}{l}\text { Se tuvo en cuenta la amistad de la alumna con sus tres mejores } \\
\text { amigos a quien ella nominó, sus tres amigos recíprocos y sus tres } \\
\text { amigos no recíprocos, relacionándolo con el número de pasos } \\
\text { dados por día medidos mediante podómetro. }\end{array}$} \\
\hline $\begin{array}{l}\text { Principales } \\
\text { resultados }\end{array}$ & \multicolumn{3}{|c|}{$\begin{array}{l}\text { Se pudo ver que cuando la amistad es recíproca, hay una fuerte } \\
\text { relación entre la actividad física de la adolecente con sus amigos en } \\
\text { comparación con las amistades no recíprocas. }\end{array}$} \\
\hline
\end{tabular}

\begin{tabular}{|c|c|c|c|}
\hline Artículo & \multicolumn{3}{|c|}{$\begin{array}{l}\text { Better with a buddy: influence of best friends on children's physical } \\
\text { activity. }\end{array}$} \\
\hline Autor & \multicolumn{3}{|c|}{ Jago et al ${ }^{(25)}$} \\
\hline Revista & \multicolumn{3}{|c|}{ Medicine \& Science in Sports \& Exercise. } \\
\hline $\begin{array}{l}\text { Año de } \\
\text { publicación }\end{array}$ & 2011 & Tipo de estudio & Transversal. \\
\hline $\begin{array}{l}\text { Variables } \\
\text { estudiadas }\end{array}$ & \multicolumn{3}{|c|}{$\begin{array}{l}\text { Con el uso de acelerómetros se midió la carga y la intensidad de la } \\
\text { actividad física (Mean accelerometer counts per minute (CPM) and } \\
\text { mean minutes of moderate to vigorous intensity physical activity per }\end{array}$} \\
\hline
\end{tabular}




\begin{tabular}{|l|l|}
\hline & $\begin{array}{l}\text { day (MVPA)) y se relacionó con el niño y sus amigos dentro de la } \\
\text { escuela, preguntando a su vez sobre aspectos en la realización de } \\
\text { actividad física. También se recogió peso y talla de cada uno para la } \\
\text { estimación del IMC. }\end{array}$ \\
\hline $\begin{array}{l}\text { Principales } \\
\text { resultados }\end{array}$ & $\begin{array}{l}\text { Los resultados obtenidos indican que, tanto en adolescentes } \\
\text { femeninas como en masculinos, ocurre que si realizan actividad } \\
\text { física con sus mejores amigos, mejoran sus niveles de actividad } \\
\text { física. }\end{array}$ \\
\hline
\end{tabular}

\begin{tabular}{|l|l|l|}
\hline Artículo & $\begin{array}{l}\text { How physical activity shapes, and is shaped by, adolescent } \\
\text { friendships. }\end{array}$ \\
\hline Autor & De la Haye et al ${ }^{(26)}$. \\
\hline Revista & Social Science \& Medicine. \\
\hline $\begin{array}{l}\text { Año de } \\
\text { publicación }\end{array}$ & 2011 & Tipo de estudio $\quad$ Longitudinal. \\
\hline $\begin{array}{l}\text { Variables } \\
\text { estudiadas }\end{array}$ & $\begin{array}{l}\text { Se preguntó sobre los lazos de amistad dentro de la clase y se } \\
\text { relacionó con la participación en la actividad física (frecuencia y } \\
\text { cantidad) y los conocimientos en la materia (actitud hacia el } \\
\text { deporte, normas de los compañeros, control percibido, } \\
\text { autoeficacia e intención de realización de ejercicio físico). }\end{array}$ \\
\hline $\begin{array}{l}\text { Principales } \\
\text { resultados }\end{array}$ & $\begin{array}{l}\text { Se llegó a la conclusión de que los adolescentes tienden a formar } \\
\text { lazos de amistad con actitudes similares hacia la actividad física y } \\
\text { con posterioridad, es decir, después de elegirse como amigos, } \\
\text { imitan los comportamientos de sus iguales. }\end{array}$ \\
\hline
\end{tabular}

\begin{tabular}{|c|c|c|c|}
\hline Artículo & \multicolumn{3}{|c|}{$\begin{array}{l}\text { School-based friendship networks and children's physical activity } \\
\text { A spacial analytical approach. }\end{array}$} \\
\hline Autor & \multicolumn{3}{|c|}{ Macdonald Wallis et al ${ }^{(27)}$} \\
\hline Revista & \multicolumn{3}{|c|}{ Social Science \& Medicine. } \\
\hline $\begin{array}{l}\text { Año de } \\
\text { publicación }\end{array}$ & 2011 & Tipo de estudio & Transversal. \\
\hline $\begin{array}{l}\text { Variables } \\
\text { estudiadas }\end{array}$ & \multicolumn{3}{|c|}{$\begin{array}{l}\text { Mediante acelerómetro se midió la carga y la intensidad de la } \\
\text { actividad física (CPM y MVPA) y se preguntó sobre la autoeficacia } \\
\text { en la realización de actividad física. Se recogió el peso y la talla } \\
\text { para la determinación del IMC de cada individuo para luego } \\
\text { buscar la asociación entre el niño y sus cuatro mejores amigos } \\
\text { con respecto a su ubicación geográfica. }\end{array}$} \\
\hline $\begin{array}{l}\text { Principales } \\
\text { resultados }\end{array}$ & \multicolumn{3}{|c|}{$\begin{array}{l}\text { La cantidad de actividad física que realiza un adolescente está } \\
\text { relacionada positiva y significativamente correlacionada con lo } \\
\text { realizado con sus amigos inmediatos y con los secundarios, } \\
\text { separados a un grado de distancia. }\end{array}$} \\
\hline
\end{tabular}

Fuente: elaboración propia. 


\section{DISCUSIÓN}

\section{Las relaciones sociales y su influencia en la ingesta de alimentos}

Los hábitos alimenticios son uno de los factores determinantes, junto con la actividad física, que contribuye, en mayor medida, a la aparición de sobrepeso y obesidad. Los adolescentes, a través de su red de amigos y colegas, pueden estar influenciados en cuanto a la cantidad y al tipo de alimentos que ingieren.

Se parte de la base de que una buena alimentación proporciona a los niños un desarrollo adecuado y una garantía de salud a largo plazo, tanto durante la niñez como en la adolescencia. A su vez, se considera que unos buenos hábitos de alimentación así como seguir una dieta equilibrada son la clave para alcanzar el objetivo de salud ideal para el adolescente ${ }^{(28)}$. En la actualidad estos hábitos alimenticios de los jóvenes están cambiando, siendo sustituidos algunos de los comportamientos saludables por otros más nocivos influenciados por la red social que forma parte de su entorno.

La cantidad de comida que se ingiere puede estar condicionada por múltiples factores, dentro de los cuales destaca la influencia que ejerce el entorno social que rodea al individuo. En sujetos adultos, se ha podido comprobar que la gente come más cantidad de alimento si está en compañía de otros individuos, en comparación a cuando comen solos. Este hecho se debe probablemente a la extensión del tiempo a la hora de comer, resultado de la interacción social, sobre todo si están acompañados de familiares y amigos en la que se añade un ambiente de relax y desinhibición ${ }^{(29)}$. Al igual que en la etapa adulta, en el estudio de Lemung y Hillman ${ }^{\left({ }^{30}\right)}$ Ilevado a cabo con niños en edad preescolar, se pudo demostrar una asociación entre el tamaño del grupo y la cantidad de comida ingerida. Concretamente, el grupo con más niños ingería más cantidad, más rápidamente y durante más tiempo, mostrando menos interacción entre ellos en comparación con el grupo más pequeño.

En cambio en la etapa de la adolescencia, cobra más importancia la naturaleza del lazo con quien se comparte la comida.

La cantidad de comida ingerida varía dependiendo de si la compañía a la hora de comer es familiar o no. Péneau, en su estudio pudo comprobar que se comen menos cantidades en compañía no familiar (individuos de la misma edad, mismo vecindario que podrían ser compañeros de clase) que si lo hacen solos, viendo la televisión o escuchando música ${ }^{(20)}$. En esta misma línea y probablemente debido a la estigmatización social y al querer causar una buena impresión se producen efectos similares de variabilidad dependiendo del estado de peso del niño y de sus colegas. De esta forma, al igual que el efecto producido en adolescentes, se ha podido comprobar cómo infantes a estudio de entre 6 y 10 años con sobrepeso comen más cuando están solos que cuando están con desconocidos, a diferencia del individuo con peso normal, que come igual independientemente del lazo de unión ${ }^{(31)}$.

Con respecto al tipo o calidad de comida, De la Haye ${ }^{(21)}$ encontró en su estudio resultados interesantes con respecto al alumno popular. Pudo comprobar que los adolescentes masculinos quienes consumían snacks calóricos tendían a ser más populares que si comían otros tipos de alimentos más saludables. Este efecto no solamente se dio con la calidad en la comida sino que también se encontró con respecto a otros comportamientos saludables, como el ejercicio físico. Se observó, 
pues, que incluso el liderazgo de un adolescente puede aumentar dependiendo de lo que coma, y por lo tanto, convertirse en un modelo para los demás y puso de relieve el papel que ocupan estos alumnos como fuente de influencia social sobre todo dentro de sus redes de amistad.

La red de amistad parece influir también en el tipo de comida, consumiendo determinados productos tales como la comida rápida, los snacks dulces y salados, las bebidas altamente calóricas, frutas y verduras, hábito del desayuno, etc. ${ }^{(21-23)}$. Ali et al (22) encontraron en su estudio una asociación positiva entre el individuo y sus amigos en relación a la frecuentación de restaurantes de comida rápida al igual que De la Haye et al. ${ }^{(21)}$, encontraron en esta misma línea, correlación significativa entre el consumo de snacks y comida rápida. Bruening et al. ${ }^{(23)}$ clasificaron la amistad como "grupo de amigos" y "mejores amigos" viendo que podría haber diferentes influencias en función de la intensidad del lazo de unión. Efectivamente se encontró asociación significativa entre el grupo de "mejores amigos" y los adolescentes en cuanto a la ingesta del desayuno, de cereales de grano entero, de productos lácteos y en el consumo de verduras, aunque reconoce poca significancia o más bien nula si nos referimos a las frutas. ${ }^{(23)}$.

Por todo lo expuesto, se pone de manifiesto la importancia del entorno social sobre la cantidad y la calidad de comida ingerida por los individuos. Son importantes los amigos, la familia, los colegas, etc., sin embargo, hay una carencia de estudios en adolescentes y con métodos específicos para medir ese entorno social, por lo que el ARS podría ser una herramienta útil para futuras investigaciones en este contexto.

\section{La influencia del entorno social en la realización de la actividad física en el adolescente}

La literatura que se muestra en este apartado demuestra que el entorno social que rodea al adolescente ejerce un efecto positivo o negativo en la realización de actividad física y deportes activos. Es importante señalar que en relación con la actividad física, el tiempo dedicado al deporte se ha visto sustituido en los últimos años por tiempo dedicado a dispositivos de pantalla como la televisión, el ordenador, video-consolas y móvil (32). Rodríguez-Hernández et al., en su estudio de escolares de entre 4 y 15 años, demostró que la obesidad y el sedentarismo son factores asociados en gran medida a peores estados de salud mental, asociando la conducta sedentaria con problemas de relaciones sociales con compañeros y otros problemas de conducta, relacionando el peso saludable con el bienestar psicológico y vinculando la práctica de actividad física con un buen estado de salud infantil ${ }^{(33)}$.

En este sentido, las recomendaciones aconsejan que los niños y adolescentes entre 6 y 17 años, deberían de realizar como mínimo 60 minutos al día de actividad física moderada-intensa de carácter aeróbico ("moderate-to-vigorous physical activity" (MVPA)) y al menos tres veces por semana actividades dirigidas a fortalecer los músculos y los huesos. En la misma línea, las recomendaciones de tiempo utilizado en actividades sedentarias no deben sobrepasar las 2 horas diarias ${ }^{(34)}$. Relacionado con estos datos y como cifra realmente preocupante Martínez-López EJ et al. aportan datos en la población española en donde se refleja que el $49 \%$ de la población encuestada de 12 a 16 años manifiesta superar las dos horas diarias ante la televisión al igual que el $40 \%$ refiere evitar los desplazamientos caminando ${ }^{(35)}$. 
En esta misma línea, y desde la perspectiva de redes de relaciones, los lectores se pueden preguntar cómo los adolescentes ejercen una capacidad de influencia entre ellos con respecto al ejercicio y deporte activo así como los hábitos sedentarios.

Al igual que en el comportamiento a la hora de ingerir alimentos, el grado y la reciprocidad de amistad puede influenciar la actividad física y deportiva del adolescente de distinta forma. Se entiende por amistades recíprocas la situación en la que dos personas se perciben como amigos, y amistades no recíprocas cuando una de las dos partes no percibe a la otra como tal ${ }^{(24)}$. Schofield et al. en su estudio, analizaron la amistad en cuanto a esta variable y pudieron demostrar que la reciprocidad de la amistad es un importante factor a considerar en el estudio de los niveles de actividad física de adolescentes femeninas. Se pudo comprobar que la cantidad de ejercicio que hacían las jóvenes variaba dependiendo de la naturaleza de la amistad de las amigas nominadas, siendo la amistad recíproca la que podría ejercer un mayor grado de influencia a la hora de modificar un comportamiento ${ }^{(24)}$.

En concordancia, otros autores han obtenido similares resultados en la adolescencia temprana diferenciando por género, encontrando que los chicos quienes tienen mejores amigos activos emplean más minutos al día en MVPA, participando en gran cantidad de ejercicio físico y que las chicas quienes frecuentemente realizan actividad física con sus mejores amigas tienen mejores niveles de actividad física, si las comparamos con las chicas que lo hacen menos frecuentemente ${ }^{(25)}$.

Hay estudios que van más allá intentando saber el porqué de esta elección. En este sentido, de la Haye se planteó como objetivo el investigar si son las personas las que seleccionan a los amigos con comportamientos de actividad física similares, o si por el contrario es la influencia de los amigos lo que provoca cambios en la actividad física de las personas. Con su trabajo se llegó a la conclusión de que los niños y adolescentes eran más propensos a desarrollar amistades con niños con comportamientos de actividad física similares, y que con posterioridad, es decir, después de haberse elegido como amigos, tendían a imitar los comportamientos entre sus iguales ${ }^{(26)}$.

Por otro lado, no sólo los mejores amigos ejercen influencia en los adolescentes. El grupo entero de amigos con quien los adolescentes conviven, parece generar similares efectos con respecto a la actividad física y comportamiento sedentario. En este sentido, Ali et al. en su estudio ${ }^{(22)}$, valoran estas variables encontrando asociación entre los jóvenes y su grupo de amigos en la realización de deporte activo y ejercicio regular. Macdonald-Wallis et al. van más allá encontrando en su trabajo que no solamente influyen los mejores amigos y los compañeros más cercanos sino que también encuentra una correlación en el comportamiento entre jóvenes con más distancia de amistad, es decir, saltándose un grado de amistad correspondiéndose con los amigos de mis amigos ${ }^{(27)}$.

Con respecto a los hábitos sedentarios, los hallazgos en cuanto a si influye la amistad en el contagio de determinados comportamientos son algo más dispares. Los mismos autores ya vistos analizando variables sedentarias, no han encontrado correlación en hábitos poco activos como por ejemplo ver la televisión ${ }^{(22)}$. La interpretación que daban los autores a estos resultados era que se debía a la realización de esta práctica en sus domicilios, no siendo habitualmente un hábito compartido con sus amigos $^{(22)}$. 
En esta misma línea, otros autores tampoco ven claro el contagio de comportamiento entre amigos en determinados hábitos sedentarios. Se ha encontrado que en cuestión de género, se pueden encontrar por un lado diferencias entre chicos y chicas en la utilización de dispositivos de pantalla en su tiempo libre. Dentro del grupo de los varones hubo diferencias entre el individuo y sus amigos en 2 de las 3 redes estudiadas, al contrario que en las redes femeninas en las que se pudo encontrar un comportamiento similar en esta misma variable ${ }^{(21)}$.

\section{CONCLUSIONES}

La red de amistad y en general el grupo de colegas y compañeros de clase de los que se acompaña el adolescente ejerce una influencia que puede modificar tanto comportamientos saludables como no saludables en materia relacionada con el estudio de la obesidad. El abordar el problema desde una perspectiva de Análisis de Redes Sociales puede aportar a las estrategias de intervención, un frente nuevo de actuación que contribuya a paliar esta epidemia mundial. Se demandan en este sentido soluciones multifactoriales llevadas a cabo por personal multidisciplinar que estudien al joven dentro de la comunidad y no de forma individual. El estudio de la estructura social en la que está inmerso el individuo, sus lazos de unión con el resto, así como el liderazgo del grupo, juegan un papel fundamental para poder afrontar la obesidad del adolescente.

\section{REFERENCIAS}

1.Organización Mundial de la Salud. Estrategia mundial sobre régimen alimentario, actividad física y salud. Sobrepeso y obesidad. Causas. Diponible en: http://www.who.int/dietphysicalactivity/childhood why/es/. [Consultado el 7 de mayo de 2014].

2. Koehly LM, Loscalzo A. Adolescent obesity and social networks. Prev Chronic Dis 2009; 6(3): A99.

3. Organización Mundial de la Salud. Diet, nutrition and the prevention of chronic diseases. Report of a Joint FAO/WHO Expert consultation. Ginebra: OMS; 2003. WHO Technical report series 916.

4. Thompson-Reid PE. Engaging and mobilizing community members to prevent obesity among adolescents. Prev Chronic Dis 2009; 6 (3): A100. Disponible en http://www.cdc.gov/pcd/issues/2009/jul/08_0259.htm . Consultado el [8 de mayo de 2014 ]

5. European Commission. Horizon 2020. The EU Framework Programme for Research and Innovation.. Disponible en: http://ec.europa.eu/programmes/horizon2020/,http://www.eshorizonte2020.es/.

[Consultado el 1 de abril de 2014]

6. Christakis N, Fowler J. The spread of obesity in a large social network over 32 years. N Engl J Med 2007; 357: 370-9.

7. Jiménez C, Gálvez E, Colomer T. Autoimagen corporal, comportamiento alimentario y estilo de vida en adolescentes. Metas de enfermería 2011; 14 (3): 69-75.

8. Esposito L, Fisher JO, Mennella JA, Hoelscher DM, Huang TT. Developmental perspectives on nutrition and obesity from gestation to adolescence. Prev Chronic Dis 2009; 6 (3): A94. Disponible en http://www.cdc.gov/pcd/issues/2009/ jul/09_0014.htm . Consultado el [9 de julio de 2014].

9. Marqués MP, Fernández R, Cabrera A, Muñoz-Doyagüe MF, Llopis J, Arias N. La sostenibilidad del sistema sanitario desde una perspectiva de redes: Una propuesta 
para la promoción de los hábitos saludables y el apoyo social. Rev Esp Salud Pública 2013; 87 (4): 307-15.

10. Salvy SJ, de la Haye K, Bowker J, Hermans CJR. Influenceof peers and friends on children's and adolescent's eating and activity behaviors. Physiol Behav 2012; 106:369-78

11. Brass DJ, Galaskiewicz, Greve HR, Tsai W. Taking stock of networks and organizations: A multilevel perspective. Acad Manage J 2004; 47 (6): 795-817.

12. Wasserman S, Faust K. Social network analysis: Methods and applications, Cambridge: Cambridge University Press; 2007.

13. Hollm-Delgado MG. Molecular epidemiology of tuberculosis transmission: contextualizing the evidence through social network theory. Soc Sci Med 2009; 69:747-53.

14. Gayen K, Reaside R. Social networks and contracepcion practice of women in rural Bangladesh. Soc Sci Med 2010; 71: 1584-92.

15. Schneider J, McFadden R, Laumann E, Kumar P, Gandham S, Oruganti G. Candidate change agent identification among men at risk for VIH infection. Soc Sci Med 2012; 75: 1192-201.

16. Strully K, Fowler JH, Joanne M, Emelia M, Levy BD, Christakis N. Aspirin use and cardiovascular events in social networks. Soc Sci Med 2012; 74: 1125-29.

17. Mulassi AH, Borracci RA, Calderón JGE, Vinay P, Mulassi M. Redes sociales de tabaquismo, consumo de alcohol y obesidad e adolescentes escolarizados de la ciudad de Lobos. Arch Argent Pediatr 2012; 110(6): 474-482.

18. Valente TW, Fujimoto K, Chou CP, Spruijt-Metz D. Adolescents affliations and adiposity: A social network analysis of friendship and obesity. Journal of Adolescent Health 2009; 45: 202-204.

19. McPherson, J.M.; Smith-Lovin, L.; Cook, J. Birds of a feather: homophily in social networks. Annual Review of Sociology 2001; 27:415-444

20. Péneau S, Mekhmoukh A, Chapelot D, Dalix A, Airinei G, Hercberg S, et al. Influence of environmental factors on food intake and choice of beverage during meals in teenagers: a laboratory study. Br J Nutr 2009; 102:1854-9.

21. De la Haye K, Robins G, Mohr P, Wilson C. Obesity-related behaviors in adolescent friendship networks. Soc Networks 2010; 32: 161-67.

22. Ali MM, Amialchuk A, Heiland FW. Weight-related behavior among adolescents: the role of peer effects. PLoS One. 2011;6(6):e21179.

23. Bruening M, Eisenberg M, MacLehose R, Nanney MS, Story M, Neumark-Sztainer D. Relationship between adolescent's and their friend's eating behaviors: breakfast, fruit, vegetable, whole grain and dairy intake. Journal of the academy of nutrition and dietetics 2012; 112(10): 1608-13.

24. Schofield L, Mummery WK, Schofield G, Hopkins W. The association of objectively determined physical activity behavior among adolescent female friends. Res $Q$ Exerc Sport. 2007;78(2):9-15.

25. Jago R, Macdonald Wallis K, Thompson JL, Page AS, Brockman R, Fox KR. Better with a buddy: influence of best friends on children's physical activity. Med Sci Sports Exerc. 2011;43(2):259-65.

26. De la Haye K, Robins $G$, Mohr $P$, Wilson C. How physical activity shapes, and is shaped by, adolescent friendships. Soc Sci Med. 2011;73(5):719-28

27. Macdonald-Wallis K, Jago R, Page AS, Brockman R, Thompson JL. School-based friendship networks and children's physical activity: A spacial analytical approach. Soc Sci Med. 2011;73:6-12.

28. Estrategia NAOS. Disponible en: http://www.naos.aesan.msssi.gob.es/csym/nutricion saludable/recomendaciones/. [Consultado el 13 de abril de 2014]. 
29. De Castro JM. Family and friends produce greater social facilitation of food intake than other companions. Physiol Behav 1994; 56: 445-55.

30. Lemung JC, Hillman KH. Eating in larger groups increases food consumption. Arch Dis Child 2007;92:384-837.

31. Salvy SJ, Coelho JS, Kieffer E, Epstein LH. Effects of social contexts on overweight and normal-weight children's food intake. Physiol Behav 2007; 92(5):8406.

32. Martí S. Actividad física, sedentarismo frente a pantallas y su relación en adolescentes [tesis]. Las Palmas de Gran Canaria: Universidad de las Palmas de Gran Canaria; 2011.

33. Rodríguez-Hernández A, de la Cruz-Sánchez E, Feu S, Martínez-Santos R. Sedentarismo, obesidad y salud mental en la población española de 4 a 15 años de edad. Rev Esp Salud Pública 2011; 85: 373-382.

34. Moreno LA, Gracia-Marco L y Comité de Nutrición de la Asociación Española de Pediatría. Prevención de la obesidad desde la actividad física: del discurso teórico a la práctica. An Pediatr (Barc) 2012; 77(2): 136.e1-136.e6.

35. Martínez-López EJ, Cachón J, Moral JE. Influences of the school and family and family context in the adolescent's physical activity. Special attention to the obese pupil. J Sport Health Res 2009; 1(1): 26-45.

Recibido: 14 de diciembre 2014; Aceptado: 9 de febrero 2015

ISSN 1695-6141

(C) COPYRIGHT Servicio de Publicaciones - Universidad de Murcia 